\title{
Article \\ Nonequivalent After-Effects of Alternating Current Stimulation on Motor Cortex Oscillation and Inhibition: Simulation and Experimental Study
}

\author{
Makoto Suzuki ${ }^{1, *}$, Satoshi Tanaka ${ }^{2}$, Jose Gomez-Tames ${ }^{3,4} \mathbb{D}^{\mathbb{C}}$, Takuhiro Okabe ${ }^{1} \mathbb{D}$, Kilchoon Cho $^{1}$, Naoki Iso ${ }^{1}$ \\ and Akimasa Hirata ${ }^{3,4}$ (D)
}

\section{check for}

updates

Citation: Suzuki, M.; Tanaka, S.;

Gomez-Tames, J.; Okabe, T.; Cho, K.; Iso, N.; Hirata, A. Nonequivalent After-Effects of Alternating Current Stimulation on Motor Cortex Oscillation and Inhibition:

Simulation and Experimental Study. Brain Sci. 2022, 12, 195. https:// doi.org/10.3390/brainsci12020195

Academic Editor: Moussa

Antoine Chalah

Received: 22 December 2021

Accepted: 28 January 2022

Published: 31 January 2022

Publisher's Note: MDPI stays neutral with regard to jurisdictional claims in published maps and institutional affiliations.

Copyright: (c) 2022 by the authors. Licensee MDPI, Basel, Switzerland. This article is an open access article distributed under the terms and conditions of the Creative Commons Attribution (CC BY) license (https:/ / creativecommons.org/licenses/by/ $4.0 /)$.
1 Faculty of Health Sciences, Tokyo Kasei University, 2-15-1 Inariyama, Sayama 350-1398, Saitama, Japan; okabe-t@tokyo-kasei.ac.jp (T.O.); cho-k@tokyo-kasei.ac.jp (K.C.); iso-n@tokyo-kasei.ac.jp (N.I.)

2 Laboratory of Psychology, Hamamatsu University School of Medicine, 1-20-1 Handayama, Higashi-ku, Hamamatsu 431-3192, Shizuoka, Japan; tanakas@hama-med.ac.jp

3 Department of Electrical and Mechanical Engineering, Nagoya Institute of Technology, Gokiso-cho, Showa-ku, Nagoya 466-8555, Aichi, Japan; jgomez@nitech.ac.jp (J.G.-T.); ahirata@nitech.ac.jp (A.H.)

4 Center of Biomedical Physics and Information Technology, Nagoya Institute of Technology, Gokiso-cho, Showa-ku, Nagoya 466-8555, Aichi, Japan

* Correspondence: suzuki-mak@tokyo-kasei.ac.jp; Tel.: +81-42-955-6074
Abstract: The effects of transcranial alternating current stimulation (tACS) frequency on brain oscillations and cortical excitability are still controversial. Therefore, this study investigated how different tACS frequencies differentially modulate cortical oscillation and inhibition. To do so, we first determined the optimal positioning of tACS electrodes through an electric field simulation constructed from magnetic resonance images. Seven electrode configurations were tested on the electric field of the precentral gyrus (hand motor area). We determined that the Cz-CP1 configuration was optimal, as it resulted in higher electric field values and minimized the intra-individual differences in the electric field. Therefore, tACS was delivered to the hand motor area through this arrangement at a fixed frequency of $10 \mathrm{~Hz}$ (alpha-tACS) or $20 \mathrm{~Hz}$ (beta-tACS) with a peak-to-peak amplitude of $0.6 \mathrm{~mA}$ for $20 \mathrm{~min}$. We found that alpha- and beta-tACS resulted in larger alpha and beta oscillations, respectively, compared with the oscillations observed after sham-tACS. In addition, alpha- and beta-tACS decreased the amplitudes of conditioned motor evoked potentials and increased alpha and beta activity, respectively. Correspondingly, alpha- and beta-tACSs enhanced cortical inhibition. These results show that tACS frequency differentially affects motor cortex oscillation and inhibition.

Keywords: electric field simulation; oscillation; primary motor cortex; spike-timing-dependent plasticity; transcranial alternating current stimulation

\section{Introduction}

Primary motor cortex (M1) excitability is affected by the dynamic oscillations of the cerebello-thalamo-cortical network. In general, cortical excitability (CE) has been correlated with dynamic network interactions that are reflected by alpha- and beta-band oscillations [1-3], which have been associated with inhibitory $\gamma$-aminobutyric acid (GABAergic) interneurons in several cortical regions, including M1 [4-6]. Therefore, the oscillatory activity in both alpha- and beta-bands in inhibitory GABAergic interneurons modulates M1 excitability [7,8].

Recently, transcranial alternating current stimulation (tACS), which involves the noninvasive delivery of a weak alternating current to the scalp, has been used to modulate cortical oscillatory activity and excitability in a frequency-specific manner [9-13]. Such modulatory effects have been reported to occur not only during but also after stimulation [9-11]. A possible mechanism underlying tACS-induced after-effects is spike-timing-dependent plasticity (STDP) [14]. In STDP, the pre- and post-synaptic potentials resulting from the 
rhythm of electrical stimulation-derived neuronal excitation, together with the intrinsic oscillatory patterns of the potentials themselves, affect the magnitude and direction of synaptic strength, leading to synaptic long-term potentiation (LTP) or long-term depression (LTD) $[10,14,15]$. Previous studies have not only shown that alpha-tACS increased the alpha-band power of brain oscillations but also have shown that this effect outlasted the end of stimulation by $\geq 30 \mathrm{~min}$ [10-12] and that the strength of the entrained oscillatory power was positively correlated with the strength of the after-effects [16,17]. These results imply that the effects of tACS on brain oscillation and excitability may last beyond stimulation.

Several studies have suggested that alpha and beta oscillations are negatively correlated with CE $[18,19]$, and others have reported decreased corticospinal excitability after $15 \mathrm{~Hz}$ and $20 \mathrm{~Hz}$ tACS $[19,20]$. However, some studies have reported no such changes in excitability after $10 \mathrm{~Hz}$ and $20 \mathrm{~Hz}$ tACS [19,21,22], while one study reported increased corticospinal excitability after $20 \mathrm{~Hz}$ tACS [23]. Therefore, the after-effects of tACS remain controversial. One of the reasons for these inconsistencies is the inter-individual variability in electric fields, which is attributable to the different brain anatomy of each individual [24]. Electric fields are unevenly distributed on the cortex, and little is known about the optimal electrode sites and configurations for successful tACS. Accordingly, although it is known that the timing between electrical stimulation and intrinsic neuronal oscillation affects synaptic strength, the effects of frequency on cortical excitability during and after tACS are not fully understood.

To elucidate this topic, computational modeling of the head of each participant can be used to guide the optimal placement of electrodes [25-27], which could also aid in predicting the effects of stimulation [28]. Regardless, this method has limited application in the clinical setting in that it requires imaging data for each participant with neurological and mental disorders such as Alzheimer's disease and Parkinson's disease [29-33]. One promising approach to solve this is to determine the optimal electrode location for a group of participants based on the montage arrangement that delivers the highest intensity with the lowest individual variability [34,35]. The detection of optimal electrode location with highest intensity and lowest variability could be useful for clinical application by mitigating the need for individual imaging data. Therefore, the present study aimed to explore whether optimal tACS electrode montage arrangement could be obtained from individualized head models analyzed at the group level and to examine the effects of alpha- and beta-tACS delivered through such arrangement on cortical excitability. We hypothesized that, if tACS results in a frequency-specific, STDP-mediated strengthening or weakening of neuronal circuits $[14,17]$, then alpha- and beta-band oscillations would change according to tACS frequency. In particular, $10 \mathrm{~Hz}$ oscillations (i.e., alpha-band) would be synchronized with the peak phase of $10 \mathrm{~Hz}$ and $20 \mathrm{~Hz}$ tACSs (Figure 1A). Similarly, $20 \mathrm{~Hz}$ oscillations (i.e., beta-band) would be synchronized with the peak phase of $20 \mathrm{~Hz}$ tACS, as well as both the peak and trough phases of $10 \mathrm{~Hz}$ tACS (Figure 1B). Correspondingly, the magnitude of cortical inhibition would change with the increasing power of cortical oscillation resulting from different tACS frequencies. Exploring how cortical oscillations and inhibition change after alpha- and beta-tACS may contribute to our understanding of tACS-induced organizational processes.

A. Alpha band oscillation

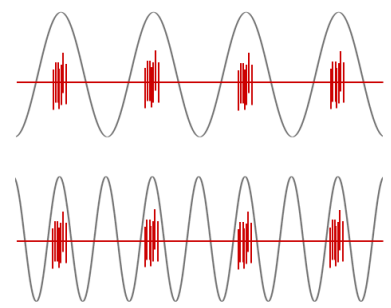

B. Beta band oscillation

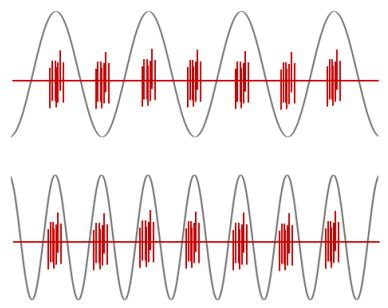

Figure 1. The hypothesized relationship between tACS frequency and neuronal activity. Gray lines denote $10 \mathrm{~Hz}$ (top trace) and $20 \mathrm{~Hz}$ (bottom trace) tACS, and red lines denote up and down states of 
neural firing. We hypothesized that (A) $10 \mathrm{~Hz}$ oscillations would be synchronized with the peak phase of $10 \mathrm{~Hz}$ and $20 \mathrm{~Hz}$ tACS, and (B) $20 \mathrm{~Hz}$ oscillations would be synchronized with both the peak and trough phases of $10 \mathrm{~Hz}$ tACS, as well as the peak phase of $20 \mathrm{~Hz}$ tACS.

\section{Materials and Methods}

\subsection{Participants}

Our experimental procedures were approved by the Research Ethics Committee of the Tokyo Kasei University (SKE2018-6) and followed the principles of the Declaration of Helsinki. All participants provided written informed consent prior to participation. In addition, all experiments were performed following the "Guidelines for TMS/tES clinical services and research through the COVID-19 pandemic" [36].

This was a single-center, single-blinded, within-participant study. The selection of the sample size was based on a desired statistical power of $80 \%$ for the detection of changes in power spectra and motor evoked potential (MEP) amplitudes, with an effect size of 0.30 and a two-sided $\alpha$-level of 0.05 . According to these parameters, G*Power 3.0 [37] yielded a sample size of 16 . Therefore, we recruited 16 healthy volunteers without neurological or psychiatric diseases who were not at risk of adverse events from transcranial magnetic stimulation (TMS) [38] and were not taking any medication. Right-handedness was confirmed with the Edinburgh Handedness Inventory [39].

\subsection{Electric Field Simulation}

A volume conductor model of the anatomical human head model was constructed using magnetic resonance imaging (MRI) images from a database of eighteen participants (all healthy males). The generated tACS electric field was conducted for the following scenarios. We considered seven tACS montages based on the International 10-20 system and anatomical structure (Figure 2A). tACS was applied with an intensity of $0.3 \mathrm{~mA}$ throughout the rubber sheets $\left(1.8 \times 1.8 \mathrm{~cm}^{2}\right)$ corresponding to a stimulation phase. We compared the normal component of the electric field values averaged over the precentral knob in the precentral area (Figure 2B) in the standard brain space among all electrode configurations for group-level analysis. Additionally, relative standard deviation (RSD) was used to quantify how much variability of the electric field was present between participants. Supplementary data presents the detailed computational model implementation.

\section{A. Electrode configurations}
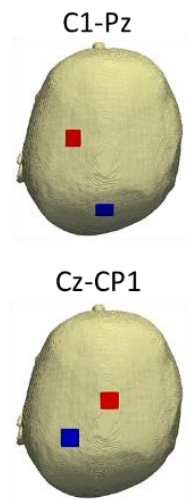
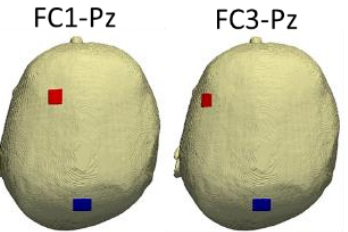

$\mathrm{C} 3-\mathrm{CPz}$
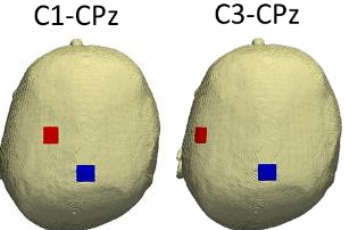

\section{B. Target region}

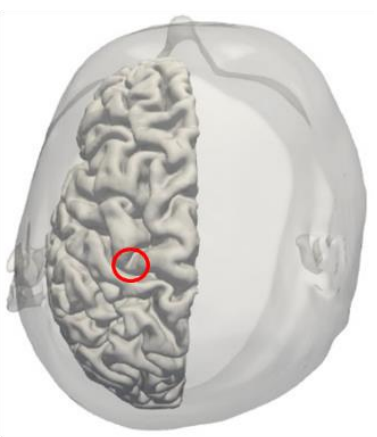

Figure 2. Electric field simulation. Seven tACS electrode configurations based on the International 10-20 system (C1-Pz, FC1-Pz, FC3-Pz, C3-Pz, Cz-CP1, C1-CPz, and C3-CPz) and anatomical structure from MRI image (A). The electric fields induced by each of the seven tACS montages were averaged on the precentral knob in the precentral area (B).

\subsection{Hotspot Detection}

Each participant was comfortably seated with their right hand resting on the testing equipment. The skin overlying the right first dorsal interosseous (FDI) muscle was 
cleaned with alcohol to reduce its electrical resistance, and recording and reference double differential surface electrodes (FAD-DEMG1, 4Assist, Tokyo, Japan) were placed over the muscle. MEPs from the FDI muscle were recorded, amplified by 100, bandpass-filtered at $10-2000 \mathrm{~Hz}$, digitized at $10 \mathrm{kHz}$ with a PowerLab system (ADInstruments, Dunedin, New Zealand), and stored in a solid-state drive.

After placing a tight-fitting cap over the participant's head, we drew intersecting nasion-inion and interaural lines on the cap with a marker pencil to localize the vertex $(\mathrm{Cz})$ in accordance with the 10-20 International System. Magstim $200^{2}$ (Magstim, Whitland, UK) stimulators were employed to deliver TMS as a monophasic current waveform via a cable to the scalp surface through a figure-of-eight coil (internal diameter of each wing: $70 \mathrm{~mm}$ ). To induce current flow in the left brain along the posterior-lateral to anterior-medial direction, we placed the coil tangentially to the scalp and held the handle so that it would point backwards and sideways, at approximately $45^{\circ}$ from the midline. As previously described $[40,41]$, we visually detected the optimal coil position to elicit maximum MEPs in the right FDI muscle ("hotspot") and marked the location with a soft-tipped pen.

\section{4. $t A C S$}

To determine the after-effects of tACS frequency on brain oscillations and cortical excitability, each participant was tested with two active (alpha- and beta-tACS) and one sham condition (Figure 3A) on three different days.

\section{A. Experimental design}

\begin{tabular}{|c|c|c|c|c|c|}
\hline RMT & MEP & EEG & tACS & EEG & MEP \\
\hline
\end{tabular}

\section{B. Electrode location}

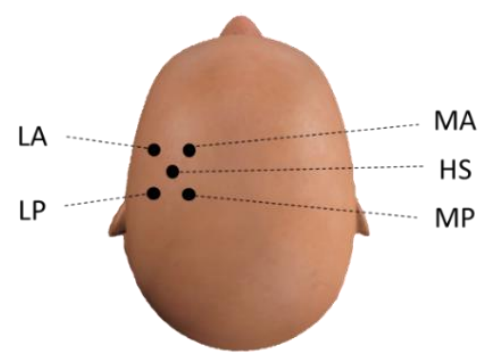

Figure 3. (A) The experimental design for testing the effects of the alpha- $(10 \mathrm{~Hz})$, beta- $(20 \mathrm{~Hz})$, and sham-tACS conditions on brain oscillations and cortical inhibition. Testing was performed on three different days. (B) Five EEG electrodes were placed at the FDI muscle hotspot (HS), and $2.5 \mathrm{~cm}$ lateral anterior (LA), medial anterior (MA), lateral posterior (LP), and medial posterior (MP) to the hotspot.

For all procedures, the participants were seated in a comfortable chair with their eyes open in a quiet room. tACS was delivered by a battery-driven current stimulator (DC Stimulator-Plus; NeuroConn, Ilmenau, Germany) through two rubber electrodes $(1.8 \times 1.8 \mathrm{~cm})$ attached to the participants' scalp. Using a conductive and adhesive paste (Ten20 Conductive Paste; Weaver and Company, Aurora, CO, USA) and a support bandage, the electrodes were placed above $\mathrm{Cz}$ and CP1, respectively, in accordance with the 10-20 International System. The Cz-CP1 montage was selected because, in our simulation, this configuration produced high current densities with low variability in the hand motor area (see Section 3). For active stimulation, tACS was delivered at a fixed frequency of $10 \mathrm{~Hz}$ (alpha-tACS) or $20 \mathrm{~Hz}$ (beta-tACS) with a peak-to-peak amplitude of $0.6 \mathrm{~mA}$ (current density: $0.093 \mathrm{~mA} / \mathrm{cm}^{2}$ ) through a current stimulator for $20 \mathrm{~min}$ [22,42]. Sham stimulation was performed at a fixed frequency of $15 \mathrm{~Hz}$ with the same intensity for $30 \mathrm{~s}$ to cause skin sensations such as tingling [43]; no current was delivered for the remaining 19 min and 
$30 \mathrm{~s}$. The order of conditions was randomized across participants, and all sessions were separated by $\geq 1$ day. Participants were blinded to the condition.

\subsection{Electroencephalography}

Electroencephalographic (EEG) data were obtained before and after tACS. The skin was prepared with alcohol, and five gold-coated active EEG electrodes were placed at the FDI muscle hotspot (HS), and $2.5 \mathrm{~cm}$ lateral anterior (LA), medial anterior (MA), lateral posterior (LP), and medial posterior (MP) to the hotspot, respectively (Figure 3B). Electrodes were mounted in an elastic cap by a holder and covered by support bandages. Electrodes were also placed above the right eye and below the left eye to record activities related to eye movements and blinking.

Participants were instructed to keep their eyes open and fixate on a $0.5 \mathrm{~cm}$ blue dot on a screen located about $100 \mathrm{~cm}$ in front of them. EEG was performed using the Polymate $\mathrm{V}$ (Miyuki Giken, Tokyo, Japan), and data were sampled at $1000 \mathrm{~Hz}$ and filtered from $0.15 \mathrm{~Hz}$ to $200 \mathrm{~Hz}$. Electrode impedance was maintained at $\leq 10 \mathrm{k} \Omega$. EEG signals were referenced to the averaged recordings of the right and left earlobes.

\subsection{Cortical Inhibition Recordings}

To measure cortical inhibition and evaluate $\mathrm{GABA}_{\mathrm{A}}$-mediated inhibitory effects, MEPs and short-interval intra-cortical inhibition (SICI) were measured before and after tACS [44,45]. The hotspot's resting motor threshold (RMT) was defined as the minimum stimulus intensity required to elicit an MEP $\geq 50 \mu \mathrm{V}$ in the relaxed FDI muscle in 5 out of 10 consecutive trials. Unconditioned MEPs for the FDI muscle were evoked at the hotspot at $120 \%$ of the RMT value. The stimulus intensity for the first conditioning pulse was set at $80 \%$ of the RMT value, and the second test pulse was administered suprathreshold at an intensity of $120 \%$ that of the RMT. A $2.5 \mathrm{~ms}$ interstimulus interval was used to test SICI $[44,45]$. Twenty trials of both unconditioned MEP and SICI measurements at a frequency of $0.2 \mathrm{~Hz}$ were recorded in random order.

\subsection{Data Analysis}

\subsubsection{EEG Data Processing}

The six EEG datasets (i.e., data collected before and after alpha-, beta-, and sham-tACS) were each split into 180 non-overlapping 1 s epochs. All epochs were visually inspected, and those containing eye blinks or muscle movement artifacts were excluded. After artifact rejection, the fast Fourier transform was applied for frequencies between 0 and $40 \mathrm{~Hz}$ ( $1 \mathrm{~Hz}$ resolution) for individual epochs using a Hanning window. After logarithmically transforming and averaging the power values of the five electrodes, frequency bands of interest were selected in the alpha $(10 \pm 1 \mathrm{~Hz})$ and beta $(20 \pm 1 \mathrm{~Hz})$ ranges, taking into account the tACS frequencies.

In order to conduct a proper comparison for differences in power spectra between tACS frequency conditions, normality testing using the Kolmogorov-Smirnov test was used. Based on the result of the Kolmogorov-Smirnov testing, either parametric two-way repeated measures analysis of variance (ANOVA) or nonparametric Friedman's test was used. Additionally, for nonparametric testing, the logarithmically transformed power spectrum without normality distribution was normalized to baseline (i.e., before tACS) according to the following equation:

$$
N P(f, t)=\frac{A(f, t)-R(f)}{R(f)},
$$

where NP denotes the normalized power spectrum, $A$ represents the EEG power spectrum at time $t$ and frequency $f$ (i.e., the power spectrum of 10 and $20 \mathrm{~Hz}$ after tACS), and $R$ denotes the mean power spectrum of the baseline period, defined as the $3 \mathrm{~min}$ interval before tACS. A large positive value indicates a large increase in the EEG power spectrum from the baseline period [46]. Furthermore, post hoc analysis with parametric Bonferroni 
correction or the nonparametric Steel-Dwass test was performed to compare differences in power spectra between tACS frequency conditions.

\subsubsection{MEP Data Processing}

A previous study [47] noted that MEP amplitudes randomly fluctuate between stimuli. Therefore, peak-to-peak MEP amplitudes were evaluated for the existence of outliers through Tukey's fences, with values more than 1.5 times that of the interquartile range excluded from the datasets [48]. To increase the precision of level and slope estimations of cortical inhibition, the blank cells produced by removing the outliers were then linearly interpolated. Next, time-series analyses were conducted using the Bayesian method. The local linear trend model (LLT) assumes that both the level (Equation (3)) and slope (Equation (4)) of the trend from observational values (Equation (2)) follow Gaussian random walks. LLTs were constructed for the MEP amplitudes as follows:

$$
\begin{gathered}
y_{t}=\mu_{t}+\varepsilon_{t}, \\
\mu_{t+1}=\mu_{t}+v_{t}+\xi_{t}, \\
v_{t+1}=v_{t}+\zeta_{t},
\end{gathered}
$$

where $y_{t}$ is the observational value; $\varepsilon_{t}$ indicates random variables; $\mu_{1}$, represents the initial level; $v_{1}$ is the initial slope; and $\xi_{t}$ and $\zeta_{t}$ indicate disturbances in the level and slope, respectively [49].

After state value estimation, normality testing using Kolmogorov-Smirnov test was used. Based on the result of the Kolmogorov-Smirnov testing, either parametric two-way repeated measures ANOVA or nonparametric Friedman's test was used. For nonparametric testing, conditioned and unconditioned MEP amplitudes were normalized to the baseline data (Equation (1)). In Equation (1), NP denotes normalized MEPs, $A$ denotes MEPs at time $t$, and $R$ denotes the mean MEP of the baseline period before tACS. A great positive value indicates a large increase in MEPs compared with that in the baseline period [46]. Post hoc analysis with parametric Bonferroni correction or the nonparametric Steel-Dwass test was performed to compare differences in MEP amplitudes among the three tACS conditions.

Data analysis was conducted with EMSE (Miyuki Giken, Tokyo, Japan), the SciPy package in the Python environment (Python Software Foundation, Wilmington, DE, USA), and the R 3.4.0 software (The R Foundation, Vienna, Austria). Data are expressed as means \pm standard errors of the mean (SEM). Statistical significance was set at $p<0.05$.

\section{Results}

\subsection{Electric Felds of Cortical tACS}

Figure 4 shows the group-level electric field distribution (normal component) on standard cortical brain space for different montages. The electric field was induced in the precentral gyrus for tACS with a $3.24 \mathrm{~cm}^{2}$ rubber sheet and an intensity of $0.3 \mathrm{~mA}$ on each phase. However, the field focality was not identical between the montages. In addition, we compared the averaged electric field values in the hand knob among the montages (Table 1 and Figure 4 ). The higher averaged values corresponded to $\mathrm{Cz}-\mathrm{CP} 1$ as $0.12 \mathrm{~V} / \mathrm{m}$ $(\min =0.04 \mathrm{~V} / \mathrm{m}, \max =0.22 \mathrm{~V} / \mathrm{m})$. Additionally, we found less variability in the induced electric field among participants for the Cz-CP1 ( \pm standard deviation $(S D)=0.05 \mathrm{~V} / \mathrm{m}$, relative $\mathrm{SD}=38 \%)$ and $\mathrm{FC} 1-\mathrm{Pz}( \pm \mathrm{SD}=0.04 \mathrm{~V} / \mathrm{m}$, relative $\mathrm{SD}=40 \%)$, as shown in Table 1 and Figure 4. In summary, the mean of the normal component averaged over the hand knob was higher and more stable by selecting the Cz-CP1 montage at group-level analysis, and at the same time the $\mathrm{Cz}-\mathrm{CP} 1$ montage of tACS not interfering with the EEG recording for the M1 region. 

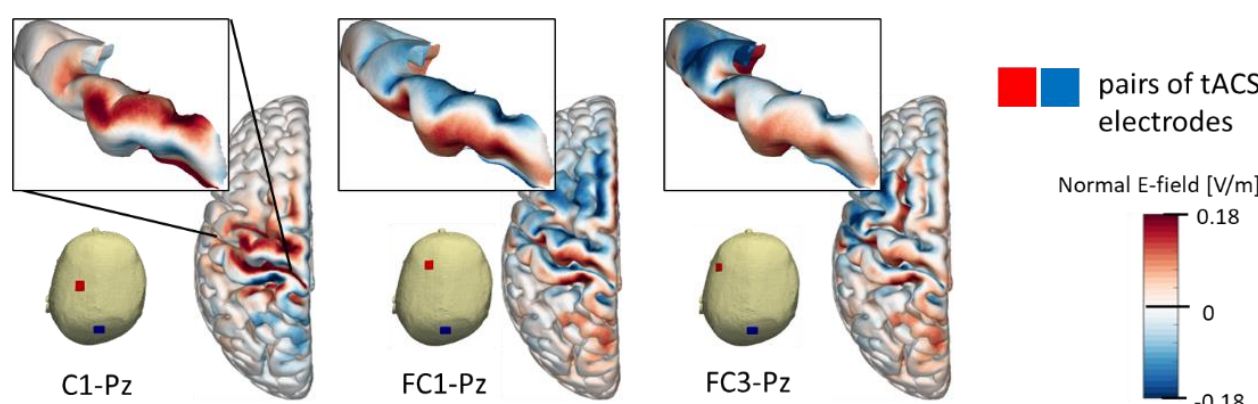

electrodes
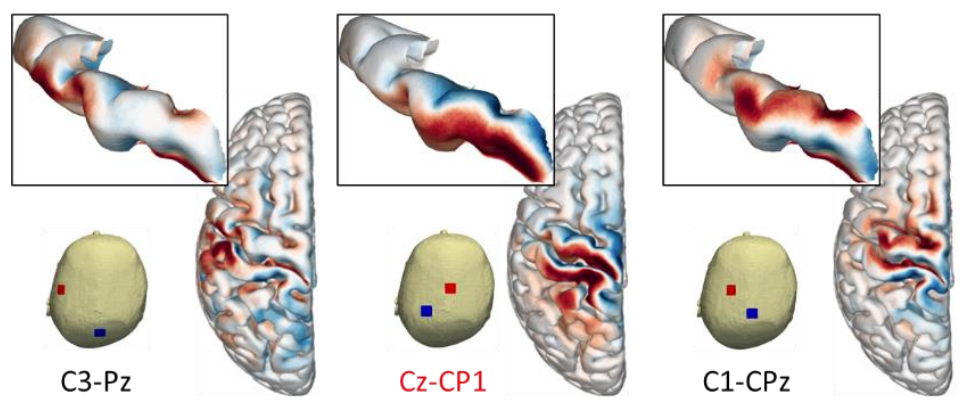

Normal E-field $[\mathrm{V} / \mathrm{m}]$

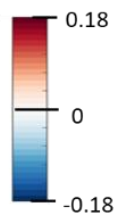

Figure 4. Normal component of the electric field (group-level analysis, $n=18$ ) during tACS in seven montages. For practical comparison, the tACS phase depicted here was chosen so that the electric field's normal component is towards the precentral wall.

Table 1. Normal component of electric field (group-level) averaged over the precentral knob.

\begin{tabular}{ccc}
\hline tACS Montage & Group-Level $(\mathbf{m V} / \mathbf{m})$ & RSD $(\%)$ \\
\hline C1-Pz & 79 & 141 \\
FC1-Pz & 107 & 40 \\
FC3-Pz & 74 & 77 \\
C3-Pz & 58 & 56 \\
Cz-CP1 & 120 & 38 \\
C1-CPz & 65 & 168 \\
C3-CPz & 59 & 58 \\
\hline
\end{tabular}

RSD, relative standard deviation; tACS, transcranial alternating current stimulation.

\subsection{Changes in Brain Oscillation and Excitation}

A total of 4 men and 12 women aged $20-40$ years ( $25.1 \pm 7.5$ years) were enrolled, and the mean laterality quotient score was $0.9(\mathrm{SD}=0.1)$. Figure 5 shows the power spectrum grand-averaged across all participants. As shown in Figure 5, the power spectrum of alphaband oscillations was increased after alpha-tACS, whereas that of beta-band oscillations was increased after beta-tACS. However, sham-tACS did not result in any changes in either power spectrum.

The Kolmogorov-Smirnov test showed that the power spectra lacked normality (alpha oscillation before and after alpha-tACS, beta-tACS, and sham-tACS: both $p<0.0001$ ). Therefore, nonparametric testing and Equation (1) was used for comparison of the power spectra of alpha and beta oscillations after alpha-, beta-, and sham-tACS treatments. The normalized power changes (i.e., the event-related synchronization (ERS) and event-related desynchronization (ERD)) in alpha-band oscillatory neural activities after alpha-, beta-, and sham-tACSs are shown in Figure 6A. The Friedman test showed a significant difference in power changes in alpha-band oscillations among alpha-, beta-, and sham-tACSs (chisquared $=16.75$, degree of freedom $=2, p=0.0002$ ). Additionally, post hoc tests showed that alpha power oscillation was greater after alpha-tACS than after sham-tACS (alpha-tACS vs. beta-tACS: $\mathrm{t}=1.37, p=0.358$; alpha-tACS vs. sham-tACS: $\mathrm{t}=3.51, p=0.001$; beta-tACS vs. sham-tACS: $\mathrm{t}=2.29, p=0.057$ ). Alpha power oscillation was greater after beta-tACS than after sham-tACS, but significance was not reached. 


\section{A. Power spectrum for alpha-tACS}

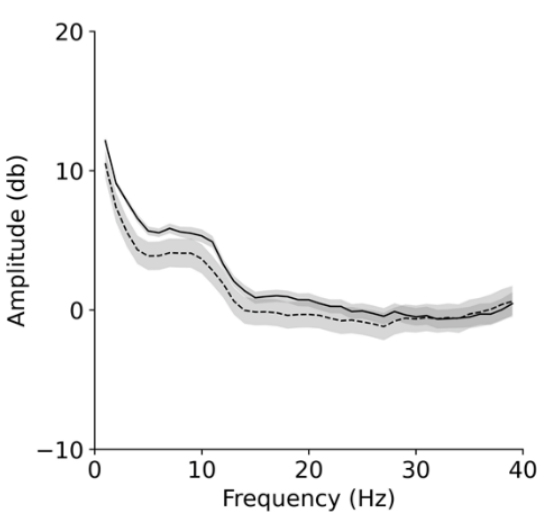

B. Power spectrum for beta-tACS

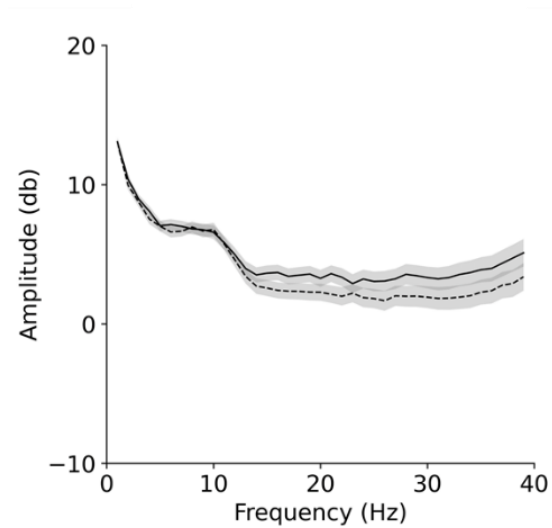

\section{Power spectrum for sham-tACS}

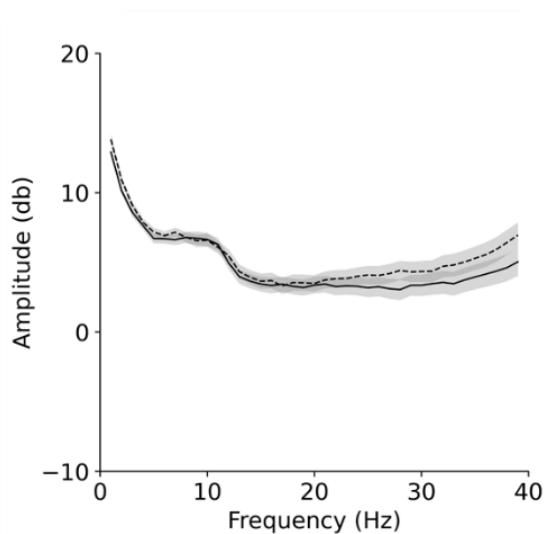

Figure 5. Grand-averaged power spectra before and after (A) alpha-, (B) beta-, and (C) sham-tACSs. Dashed and solid lines denote the power spectra before and after tACS, respectively. Shaded areas indicate the standard error of the mean. The power spectrum of alpha-band oscillation was increased after alpha-tACS, whereas beta-band oscillation was increased after beta-tACS. However, the power spectra of alpha- and beta-band oscillations were not changed by sham-tACS.

\section{A. Alpha band}

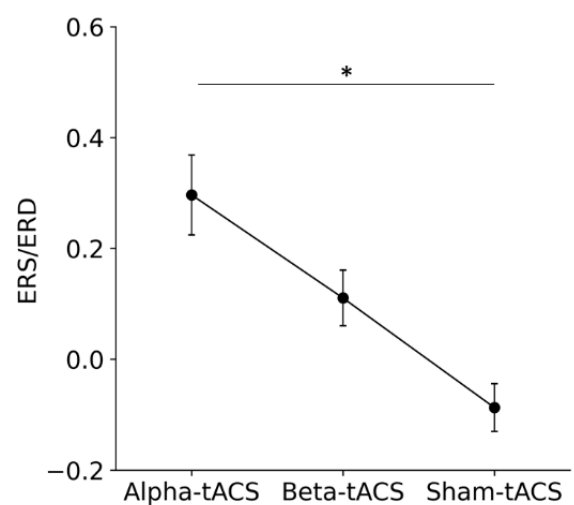

B. Beta band

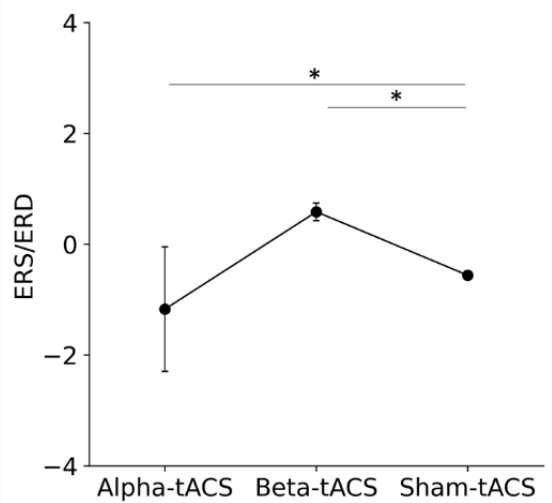

Figure 6. Normalized power changes in (A) alpha and (B) beta-oscillatory neural activity after alpha-, beta-, and sham- tACSs. Dots and error bars denote the mean and standard error of the mean, respectively. Alpha-tACS resulted in an increase in alpha power oscillations and decreased beta power oscillations, whereas beta-tACS increased beta power oscillations. *: $p<0.05$.

The ERS/ERD of beta-oscillatory neural activities after alpha-, beta-, and sham-tACSs are shown in Figure 6B. The Friedman test showed a significant difference in the ERS/ERD of beta oscillations among the alpha-, beta-, and sham-tACSs (chi-squared $=11.53$, degree of freedom $=2, p=0.003$ ). Additionally, post hoc tests showed that beta power oscillation was greater after beta-tACS than after sham-tACS (alpha-tACS vs. beta-tACS: $t=1.65$, $p=0.358$; alpha-tACS vs. sham-tACS: $\mathrm{t}=3.45, p<0.0001$; beta-tACS vs. sham-tACS: $\mathrm{t}=4.34, p=0.016)$. Moreover, beta power oscillation was lower after alpha-tACS than after sham-tACS.

The grand-averaged actual and estimated peak-to-peak MEP amplitudes according to the LLT are shown in Figure 7. The actual MEP amplitudes fluctuated randomly before and after alpha-, beta-, and sham-tACS, whereas the fluctuation in the estimated MEP amplitudes was reduced by the LLT. 


\section{A. Conditioned MEP for alpha-tACS}

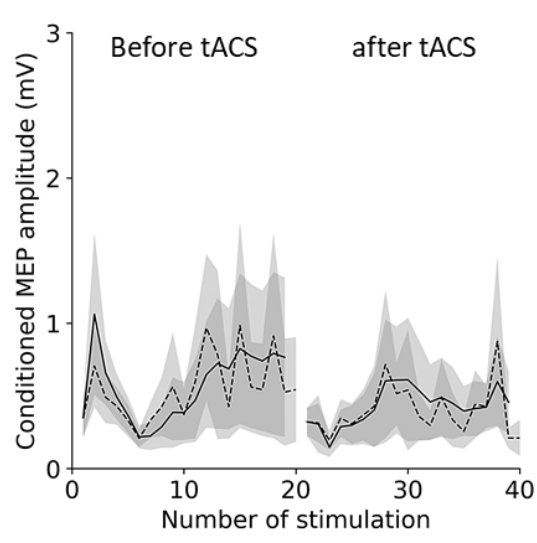

D. Unconditioned MEP for alpha-tACS

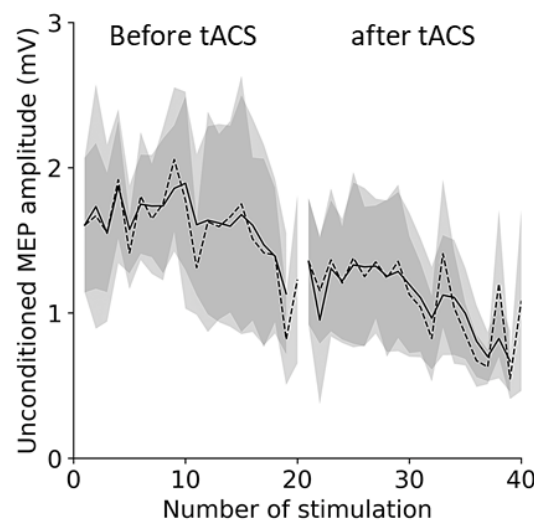

\section{B. Conditioned MEP for beta-tACS}

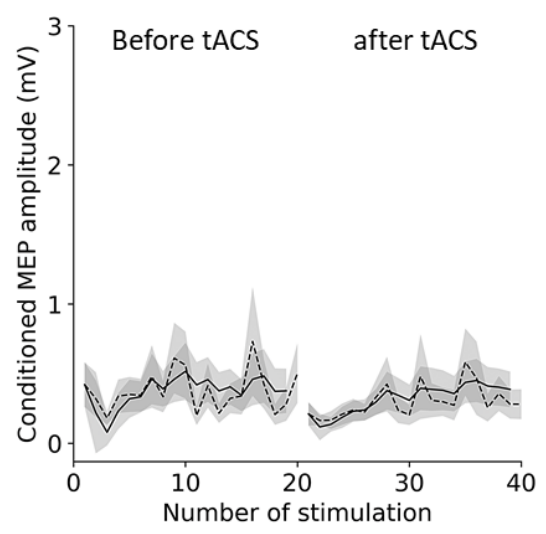

E. Unconditioned MEP for beta-tACS

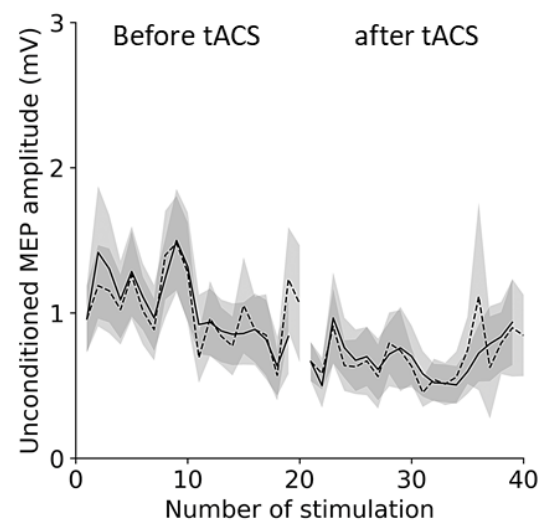

\section{Conditioned MEP for sham-tACS}

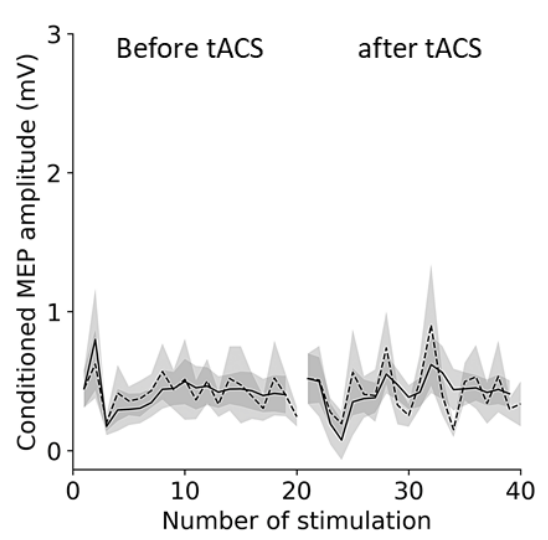

F. Unconditioned MEP for sham-tACS

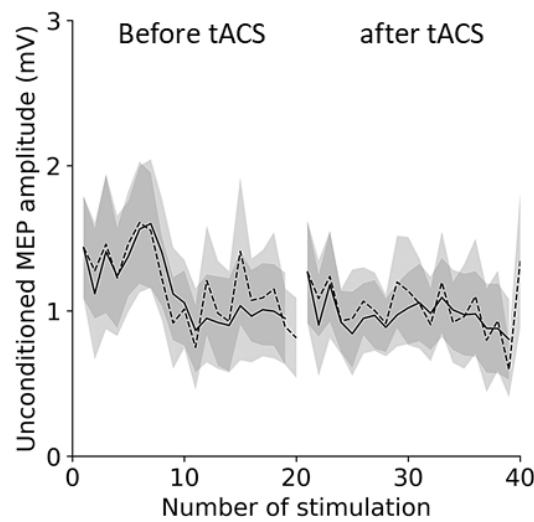

Figure 7. Grand-averaged time-series of the (A-C) conditioned and (D-F) unconditioned MEP amplitudes by the LLT model. Dashed and solid lines indicate actual and estimated MEP amplitudes, respectively. Shaded areas indicate the standard error of the mean. The actual MEP amplitudes fluctuated randomly, whereas the fluctuation of estimated MEP amplitudes was reduced by the LLT model.

The Kolmogorov-Smirnov test showed that the MEP amplitudes lacked normality (conditioned and unconditioned MEP before and after alpha-tACS, beta-tACS, and shamtACS: both $p<0.0001)$. Therefore, nonparametric testing and Equation (1) was used for comparison of the conditioned and unconditioned MEP amplitudes after alpha-, beta-, and sham-tACS treatments. The changes in the normalized conditioned MEP amplitudes after tACS are shown in Figure 8A. The Friedman test showed a significant difference in normalized condition MEP amplitudes among alpha-, beta-, and sham-tACSs (chisquared $=42.28$, degree of freedom $=2, p<0.0001$ ). Further, post hoc tests showed that the conditioned MEP amplitudes were smaller after alpha- and beta-tACS than after sham-tACS. Specifically, MEP amplitudes after alpha-tACS were smaller than those after beta-tACS (alpha-tACS vs. beta-tACS: $\mathrm{t}=2.56, p=0.029$; alpha-tACS vs. shamtACS: $\mathrm{t}=4.93, p<0.0001$; beta-tACS vs. sham-tACS: $\mathrm{t}=2.38, p=0.045$ ).

The normalized unconditioned MEP amplitudes changes after tACS are shown in Figure 8B. The Friedman test showed a significant difference among the alpha-, beta-, and sham-tACSs (chi-squared $=11.93$, degree of freedom $=2, p=0.002$ ). Further, post hoc tests showed that the unconditioned MEP amplitude was smaller after alpha- and beta-tACS than after sham-tACS (alpha-tACS vs. beta-tACS: $\mathrm{t}=1.41, p=0.338$; alpha-tACS vs. sham-tACS: $\mathrm{t}=3.80, p=0.0004$; beta-tACS vs. sham-tACS: $\mathrm{t}=2.57, p=0.028$ ). 
A. Conditioned MEP

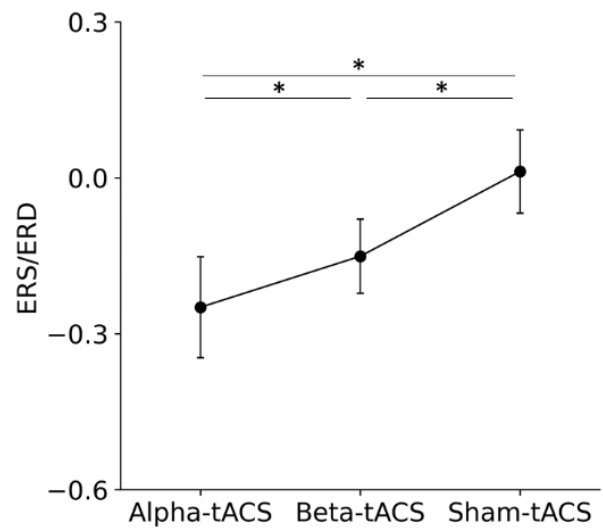

B. Unconditioned MEP

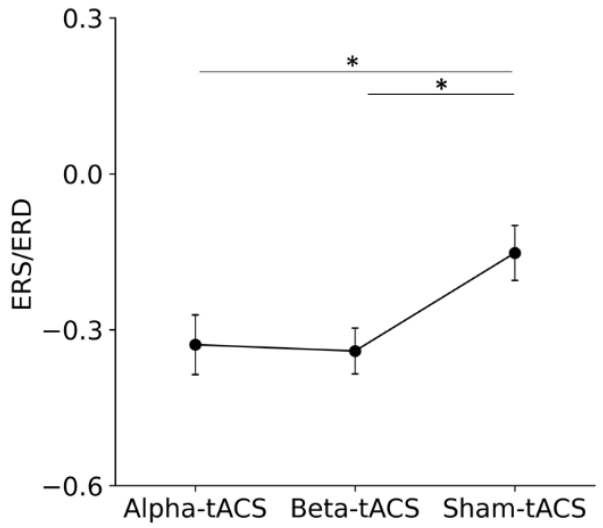

Figure 8. The normalized (A) conditioned and (B) unconditioned MEP amplitude among alpha-, beta, and sham-tACS. Dots and error bars denote the mean and standard error of the mean, respectively. Alpha- and beta-tACSs decreased both conditioned and unconditioned MEP amplitudes. ${ }^{*}: p<0.05$.

\section{Discussion}

Previous studies did not conduct a group-level evaluation of the optimal sites and montage configurations for tACS electrodes [7,13,19-23,42]. Therefore, we investigated this through a computational simulation, which showed that the tACS Cz-CP1 montage arrangement diminishes the inter-individual variability in the electric field. The electric field range for this arrangement $(0.1 \mathrm{~V} / \mathrm{m}-0.2 \mathrm{~V} / \mathrm{m})$ indicated a modulatory effect [50]. Therefore, we utilized the $\mathrm{Cz}-\mathrm{CP} 1$ montage configuration to deliver tACS. We opted for a group-level electric field analysis to maximize the electric field on the target while minimizing individual variability to determine the optimal montage applied to all participants (one-for-all) based on the International 10-20 system positioning. This is advantageous with respect to the individual-level electric field analysis, as it does not require imaging of each individual or electrode localization based on a navigation system that is not always available in clinical settings, and it did not increase the participants' time in the experiment, which was limited during the COVID-19 pandemic [36]. Therefore, our simulation based on group-level electric field analysis is advantageous for adaptation to various clinical settings, obviating the need for imaging data in the individual-level electric field analysis.

Our experimental results show that alpha- and beta-tACS result in larger alpha and beta oscillations, respectively, and they differently influence cortical inhibition. In addition, alpha-, beta-, and sham-tACS result in a stepwise decrease in conditioned MEP amplitudes. These observations show that alpha- and beta-tACS differently modulate alpha- and betaband oscillations, which, in turn, differently influence cortical inhibition. This implies that cortical oscillation and inhibition are not equally affected by alpha- and beta-tACSs. Previous studies have noted that tACS has online and offline modulatory effects during and after stimulation [9-11]. Especially, the offline effects after tACS underline its potential as a therapeutic tool because of its lasting effect beyond stimulation period.

Two possible mechanisms of tACS modulatory effect have been suggested. First, tACS directly entrains intrinsic brain oscillations [51-54]. Second, tACS leads to synaptic changes via STDP mechanisms $[10,14,15,17]$. In entrainment of brain oscillation, intrinsic brain oscillation in accordance with the external stimulation frequency will be entrained but intrinsic brain oscillation outside the stimulation frequency will not be affected [11,55]. Therefore, intrinsic alpha and beta oscillation in M1 [56,57] are externally tuned by tACS, according to the resonance-like hypothesis [23]. Adding to the entrainment mechanism, STDP could possibly explain tACS-induced after-effects [14]. Synapses are either strengthened or weakened depending on the timing of their input and output activity $[10,14,15,17]$, which might be related to the after-effects of tACS [58]. According to the STDP model, facilitatory effects on neural oscillations are expected with the synchronization between 
the peak phase of tACS and the up state of neural firing, whereas depressive effects are expected with the asynchronization between the two events (i.e., the trough phase of tACS synchronizing the up state of neural firing) $[10,14,15,17,55,58]$. In agreement with these predictions, we observed that alpha- and beta-tACS increased alpha and beta oscillatory activity, respectively, while alpha-tACS decreased beta oscillatory activity. One possible explanation for this decrement could be that both the peak and trough phases of alpha-tACS may synchronize the up state of neural firing. Previous studies have already showed an enhanced oscillatory activity after tACS $[10,14,15,17,58]$, but we found that tACS frequency influences brain oscillatory activity in a frequency-dependent manner.

Kiers et al. [47] noted that MEP amplitudes randomly fluctuated during the recording period, and Ogata et al. [18] suggested that, during the resting state, the relationship between amplitude fluctuations and cortical oscillations and inhibition is not conclusive. To solve these issues, we used the LLT model based on Bayesian estimations to examine the effects of alpha- and beta-tACS on the level and slope of cortical inhibition, thus eliminating the confounding factor of amplitude fluctuations during the resting state.

Alpha- and beta-band oscillations have been associated with the inhibitory GABAergic interneurons within M1 [4-6,59]. Accordingly, enhanced GABAergic interneuron activity plays an essential role in the modulation of M1 excitability induced by alpha- and betatACS [60]. However, a previous study found no effect of beta-tACS in SICI after TMSinduced $\mathrm{GABA}_{\mathrm{A}}$ inhibition $[7,45]$. Our findings on the alpha- and beta-tACSs effects on SICI suggest that the cortex is inhibited after stimulation. One possible explanation for this discrepancy could be that changes in the M1 post-synaptic potentials that correspond to alpha- and beta-oscillations can be altered by alpha- and beta-tACS, assuming that tACS enhances the synaptic strength of GABAergic neurons, as described by the STDP model $[10,14,15,17,58]$. Therefore, cortical inhibition might be affected by the enhancement of cortical oscillations associated with tACS frequency.

Previous work has shown that beta oscillations correlate with CE levels [61-63] and that alpha and beta oscillations inhibited MEP amplitudes [18,64-66], although a statistically significant relationship has not always been observed $[67,68]$. In addition, several studies noted that $20 \mathrm{~Hz}$ tACS increased corticospinal excitability [13,23]. However, Cappon et al. [69] and Zaghi et al. [20] observed reduced MEP amplitudes after 15 and $20 \mathrm{~Hz}$ tACS of M1, and Wach et al. [21] and Schutter and Hortensius [19] did not find any effect of 10 and $20 \mathrm{~Hz}$ tACS. However, in our study, unconditioned MEP amplitudes decreased after alpha- and beta-tACSs. Sanger et al. [70] suggested that MEP amplitude was affected not only by $\mathrm{GABA}_{\mathrm{A}}$ receptors, thus suggesting SICI, but also by excitatory glutamatergic and inhibitory $\mathrm{GABA}_{\mathrm{B}}$ receptors. However, the precise mechanism of tACS-related changes in unconditioned MEP amplitude is still unclear. Future studies should investigate changes in $\mathrm{CE}$ and inhibition related to $\mathrm{GABA}_{\mathrm{A}}, \mathrm{GABA}_{\mathrm{B}}$, and glutamatergic receptors at various tACS frequencies.

Previous studies indicate that neurological and mental diseases induce changes in brain oscillations [29-33,71,72]. In Parkinson's disease, abnormal beta activity could be related to bradykinesia [73]. Additionally, in Alzheimer's disease, abnormal alpha activity could be related to memory dysfunction [74]. The frequency-specific tACS modulatory effects for brain oscillation and inhibition could have potentially useful clinical applications. Further studies are needed to assess the tACS modulatory effect on brain oscillatory and inhibitory disorders in neurological and mental disorders such as Parkinson's disease and Alzheimer's disease.

Our study has two main limitations. First, because it was necessary to monitor tACS waves for safe and precise stimulation, we could not utilize a double-blinded design. Therefore, double-blinded studies should be conducted in the future. Second, the sample size and composition, which was estimated using $\mathrm{G}^{*}$ Power 3.0 [24], were limited as we did not consider factors such as differences in age, sex, baseline MEP sizes, or MEP's latency. In fact, concerning these two latter factors, Wiethoff et al. [75] noted that baseline MEP sizes and latency differences in MEP (anteroposterior stimulation minus latero-medial 
stimulation) result in changes in corticospinal excitability. Thus, future studies need to include a larger sample size to analyze the effects of such parameters on cortical excitability.

\section{Conclusions}

In conclusion, we found that changes in tACS frequency result in corresponding changes in alpha- and beta-band oscillations and cortical inhibition. These results imply that cortical oscillations can be differentially altered by tACS and that cortical inhibition may change according to the tACS frequency-modulated balance between alpha and beta oscillations.

Supplementary Materials: The following supporting information can be downloaded at: https: / / www.mdpi.com/article/10.3390/brainsci12020195/s1, A Electric Field.

Author Contributions: Conceptualization, M.S., S.T. and A.H.; data curation: M.S., T.O., K.C. and N.I.; formal analysis: M.S., J.G.-T. and A.H.; funding acquisition: M.S.; investigation: M.S., T.O., K.C., N.I., J.G.-T. and A.H.; methodology: M.S., S.T., J.G.-T. and A.H.; writing: M.S., J.G.-T. and A.H.; review and editing: M.S., S.T., J.G.-T., T.O., K.C., N.I. and A.H. All authors have read and agreed to the published version of the manuscript.

Funding: This work was funded by the Japan Society for the Promotion of Science, a Grant-in-Aid for Scientific Research, grant number JSPS KAKENHI $18 \mathrm{H} 03133$.

Institutional Review Board Statement: The study was conducted in accordance with the Declaration of Helsinki, and the study was approved by the Research Ethics Committee of the Tokyo Kasei University (SKE2018-6; 26 September 2020).

Informed Consent Statement: Written informed consent was obtained from all participants involved in the study.

Data Availability Statement: Raw data were generated at Tokyo Kasei University. Derived data supporting the findings of this study are available from the corresponding author, M.S.

Acknowledgments: The authors would like to acknowledge Atsushi Shirasawa, Tooru Akaike, and Yui Tatehara for their technical assistance with data collection in this study.

Conflicts of Interest: The authors declare no conflict of interest. The funders had no role in the design of the study; in the collection, analyses, or interpretation of data; in the writing of the manuscript; or in the decision to publish the results.

\section{References}

1. Gross, J.; Timmermann, L.; Kujala, J.; Dirks, M.; Schmitz, F.; Salmelin, R.; Schnitzler, A. The neural basis of intermittent motor control in humans. Proc. Natl. Acad. Sci. USA 2002, 99, 2299-2302. [CrossRef] [PubMed]

2. Pollok, B.; Gross, J.; Dirks, M.; Timmermann, L.; Schnitzler, A. The cerebral oscillatory network of voluntary tremor. J. Physiol. 2004, 554, 871-878. [CrossRef] [PubMed]

3. Pollok, B.; Gross, J.; Muller, K.; Aschersleben, G.; Schnitzler, A. The cerebral oscillatory network associated with auditorily paced finger movements. Neuroimage 2005, 24, 646-655. [CrossRef] [PubMed]

4. Baumgarten, T.J.; Oeltzschner, G.; Hoogenboom, N.; Wittsack, H.J.; Schnitzler, A.; Lange, J. Beta Peak Frequencies at Rest Correlate with Endogenous GABA+/Cr Concentrations in Sensorimotor Cortex Areas. PLoS ONE 2016, 11, e0156829. [CrossRef] [PubMed]

5. Jensen, O.; Goel, P.; Kopell, N.; Pohja, M.; Hari, R.; Ermentrout, B. On the human sensorimotor-cortex beta rhythm: Sources and modeling. Neuroimage 2005, 26, 347-355. [CrossRef] [PubMed]

6. Hall, S.D.; Stanford, I.M.; Yamawaki, N.; McAllister, C.J.; Ronnqvist, K.C.; Woodhall, G.L.; Furlong, P.L. The role of GABAergic modulation in motor function related neuronal network activity. Neuroimage 2011, 56, 1506-1510. [CrossRef]

7. Nowak, M.; Hinson, E.; van Ede, F.; Pogosyan, A.; Guerra, A.; Quinn, A.; Brown, P.; Stagg, C.J. Driving Human Motor Cortical Oscillations Leads to Behaviorally Relevant Changes in Local GABAA Inhibition: A tACS-TMS Study. J. Neurosci. 2017, 37, 4481-4492. [CrossRef]

8. Cirillo, J.; Cowie, M.J.; MacDonald, H.J.; Byblow, W.D. Response inhibition activates distinct motor cortical inhibitory processes. J. Neurophysiol. 2018, 119, 877-886. [CrossRef]

9. Feher, K.D.; Nakataki, M.; Morishima, Y. Phase-Dependent Modulation of Signal Transmission in Cortical Networks through tACS-Induced Neural Oscillations. Front. Hum. Neurosci. 2017, 11, 471. [CrossRef]

10. Zaehle, T.; Rach, S.; Herrmann, C.S. Transcranial alternating current stimulation enhances individual alpha activity in human EEG. PLoS ONE 2010, 5, e13766. [CrossRef] 
11. Neuling, T.; Rach, S.; Herrmann, C.S. Orchestrating neuronal networks: Sustained after-effects of transcranial alternating current stimulation depend upon brain states. Front. Hum. Neurosci. 2013, 7, 161. [CrossRef] [PubMed]

12. Herrmann, C.S.; Struber, D.; Helfrich, R.F.; Engel, A.K. EEG oscillations: From correlation to causality. Int. J. Psychophysiol. 2016, 103, 12-21. [CrossRef] [PubMed]

13. Nakazono, H.; Ogata, K.; Kuroda, T.; Tobimatsu, S. Phase and Frequency-Dependent Effects of Transcranial Alternating Current Stimulation on Motor Cortical Excitability. PLoS ONE 2016, 11, e0162521. [CrossRef] [PubMed]

14. Vossen, A.; Gross, J.; Thut, G. Alpha Power Increase After Transcranial Alternating Current Stimulation at Alpha Frequency (alpha-tACS) Reflects Plastic Changes Rather Than Entrainment. Brain Stimul. 2015, 8, 499-508. [CrossRef] [PubMed]

15. Tavakoli, A.V.; Yun, K. Transcranial Alternating Current Stimulation (tACS) Mechanisms and Protocols. Front. Cell Neurosci. 2017, 11, 214. [CrossRef]

16. Helfrich, R.F.; Schneider, T.R.; Rach, S.; Trautmann-Lengsfeld, S.A.; Engel, A.K.; Herrmann, C.S. Entrainment of brain oscillations by transcranial alternating current stimulation. Curr. Biol. 2014, 24, 333-339. [CrossRef]

17. Veniero, D.; Vossen, A.; Gross, J.; Thut, G. Lasting EEG/MEG Aftereffects of Rhythmic Transcranial Brain Stimulation: Level of Control Over Oscillatory Network Activity. Front. Cell Neurosci. 2015, 9, 477. [CrossRef]

18. Ogata, K.; Nakazono, H.; Uehara, T.; Tobimatsu, S. Prestimulus cortical EEG oscillations can predict the excitability of the primary motor cortex. Brain Stimul. 2019, 12, 1508-1516. [CrossRef]

19. Schutter, D.J.; Hortensius, R. Brain oscillations and frequency-dependent modulation of cortical excitability. Brain Stimul. 2011, 4, 97-103. [CrossRef]

20. Zaghi, S.; de Freitas Rezende, L.; de Oliveira, L.M.; El-Nazer, R.; Menning, S.; Tadini, L.; Fregni, F. Inhibition of motor cortex excitability with $15 \mathrm{~Hz}$ transcranial alternating current stimulation (tACS). Neurosci. Lett. 2010, 479, 211-214. [CrossRef]

21. Wach, C.; Krause, V.; Moliadze, V.; Paulus, W.; Schnitzler, A.; Pollok, B. Effects of $10 \mathrm{~Hz}$ and $20 \mathrm{~Hz}$ transcranial alternating current stimulation (tACS) on motor functions and motor cortical excitability. Behav. Brain Res. 2013, 241, 1-6. [CrossRef] [PubMed]

22. Rjosk, V.; Kaminski, E.; Hoff, M.; Gundlach, C.; Villringer, A.; Sehm, B.; Ragert, P. Transcranial Alternating Current Stimulation at Beta Frequency: Lack of Immediate Effects on Excitation and Interhemispheric Inhibition of the Human Motor Cortex. Front. Hum. Neurosci. 2016, 10, 560. [CrossRef] [PubMed]

23. Feurra, M.; Bianco, G.; Santarnecchi, E.; Del Testa, M.; Rossi, A.; Rossi, S. Frequency-dependent tuning of the human motor system induced by transcranial oscillatory potentials. J. Neurosci. 2011, 31, 12165-12170. [CrossRef] [PubMed]

24. Laakso, I.; Tanaka, S.; Mikkonen, M.; Koyama, S.; Sadato, N.; Hirata, A. Electric fields of motor and frontal tDCS in a standard brain space: A computer simulation study. Neuroimage 2016, 137, 140-151. [CrossRef] [PubMed]

25. Bikson, M.; Datta, A.; Rahman, A.; Scaturro, J. Electrode montages for tDCS and weak transcranial electrical stimulation: Role of "return" electrode's position and size. Clin. Neurophysiol. 2010, 121, 1976-1978. [CrossRef] [PubMed]

26. Opitz, A.; Yeagle, E.; Thielscher, A.; Schroeder, C.; Mehta, A.D.; Milham, M.P. On the importance of precise electrode placement for targeted transcranial electric stimulation. Neuroimage 2018, 181, 560-567. [CrossRef] [PubMed]

27. Datta, A.; Baker, J.M.; Bikson, M.; Fridriksson, J. Individualized model predicts brain current flow during transcranial directcurrent stimulation treatment in responsive stroke patient. Brain Stimul. 2011, 4, 169-174. [CrossRef] [PubMed]

28. Rawji, V.; Ciocca, M.; Zacharia, A.; Soares, D.; Truong, D.; Bikson, M.; Rothwell, J.; Bestmann, S. tDCS changes in motor excitability are specific to orientation of current flow. Brain Stimul. 2018, 11, 289-298. [CrossRef]

29. Brittain, J.S.; Probert-Smith, P.; Aziz, T.Z.; Brown, P. Tremor suppression by rhythmic transcranial current stimulation. Curr. Biol. 2013, 23, 436-440. [CrossRef]

30. Uhlhaas, P.J.; Singer, W. Neural synchrony in brain disorders: Relevance for cognitive dysfunctions and pathophysiology. Neuron 2006, 52, 155-168. [CrossRef]

31. Uhlhaas, P.J.; Singer, W. Neuronal dynamics and neuropsychiatric disorders: Toward a translational paradigm for dysfunctional large-scale networks. Neuron 2012, 75, 963-980. [CrossRef] [PubMed]

32. Latreille, V.; Carrier, J.; Gaudet-Fex, B.; Rodrigues-Brazete, J.; Panisset, M.; Chouinard, S.; Postuma, R.B.; Gagnon, J.F. Electroencephalographic prodromal markers of dementia across conscious states in Parkinson's disease. Brain 2016, 139, 1189-1199. [CrossRef] [PubMed]

33. Cozac, V.V.; Gschwandtner, U.; Hatz, F.; Hardmeier, M.; Ruegg, S.; Fuhr, P. Quantitative EEG and Cognitive Decline in Parkinson's Disease. Parkinsons Dis. 2016, 2016, 9060649. [CrossRef] [PubMed]

34. Gomez-Tames, J.; Asai, A.; Hirata, A. Significant group-level hotspots found in deep brain regions during transcranial direct current stimulation (tDCS): A computational analysis of electric fields. Clin. Neurophysiol. 2020, 131, 755-765. [CrossRef] [PubMed]

35. Soleimani, G.; Saviz, M.; Bikson, M.; Towhidkhah, F.; Kuplicki, R.; Paulus, M.P.; Ekhtiari, H. Group and individual level variations between symmetric and asymmetric DLPFC montages for tDCS over large scale brain network nodes. Sci. Rep. 2021, 11, 1271. [CrossRef]

36. Bikson, M.; Hanlon, C.A.; Woods, A.J.; Gillick, B.T.; Charvet, L.; Lamm, C.; Madeo, G.; Holczer, A.; Almeida, J.; Antal, A.; et al. Guidelines for TMS/tES clinical services and research through the COVID-19 pandemic. Brain Stimul. 2020, 13, 1124-1149. [CrossRef]

37. Faul, F.; Erdfelder, E.; Lang, A.G.; Buchner, A. G*Power 3: A flexible statistical power analysis program for the social, behavioral, and biomedical sciences. Behav. Res. Methods 2007, 39, 175-191. [CrossRef] 
38. Rossi, S.; Hallett, M.; Rossini, P.M.; Pascual-Leone, A.; Safety of TMS Consensus Group. Safety, ethical considerations, and application guidelines for the use of transcranial magnetic stimulation in clinical practice and research. Clin. Neurophysiol. 2009, 120, 2008-2039. [CrossRef]

39. Oldfield, R.C. The assessment and analysis of handedness: The Edinburgh inventory. Neuropsychologia 1971, 9, 97-113. [CrossRef]

40. Suzuki, M.; Suzuki, T.; Wang, Y.J.; Hamaguchi, T. Changes in Magnitude and Variability of Corticospinal Excitability during Rewarded Time-Sensitive Behavior. Front. Behav. Neurosci. 2019, 13, 147. [CrossRef]

41. Suzuki, M.; Suzuki, T.; Tanaka, S.; Sugawara, K.; Hamaguchi, T. Corticospinal excitability related to reciprocal muscles during the motor preparation period: Effect of movement repetition. Neuroreport 2019, 30, 856-862. [CrossRef] [PubMed]

42. Pollok, B.; Boysen, A.C.; Krause, V. The effect of transcranial alternating current stimulation (tACS) at alpha and beta frequency on motor learning. Behav. Brain Res. 2015, 293, 234-240. [CrossRef] [PubMed]

43. Kirimoto, H.; Ogata, K.; Onishi, H.; Oyama, M.; Goto, Y.; Tobimatsu, S. Transcranial direct current stimulation over the motor association cortex induces plastic changes in ipsilateral primary motor and somatosensory cortices. Clin. Neurophysiol. 2011, 122, 777-783. [CrossRef] [PubMed]

44. Amadi, U.; Allman, C.; Johansen-Berg, H.; Stagg, C.J. The Homeostatic Interaction between Anodal Transcranial Direct Current Stimulation and Motor Learning in Humans is related to GABAA Activity. Brain Stimul. 2015, 8, 898-905. [CrossRef] [PubMed]

45. Guerra, A.; Pogosyan, A.; Nowak, M.; Tan, H.; Ferreri, F.; Di Lazzaro, V.; Brown, P. Phase Dependency of the Human Primary Motor Cortex and Cholinergic Inhibition Cancelation during Beta tACS. Cereb. Cortex 2016, 26, 3977-3990. [CrossRef] [PubMed]

46. Takemi, M.; Maeda, T.; Masakado, Y.; Siebner, H.R.; Ushiba, J. Muscle-selective disinhibition of corticomotor representations using a motor imagery-based brain-computer interface. Neuroimage 2018, 183, 597-605. [CrossRef] [PubMed]

47. Kiers, L.; Cros, D.; Chiappa, K.H.; Fang, J. Variability of motor potentials evoked by transcranial magnetic stimulation. Electroencephalogr. Clin. Neurophysiol. 1993, 89, 415-423. [CrossRef]

48. van der Spoel, E.; Choi, J.; Roelfsema, F.; Cessie, S.L.; van Heemst, D.; Dekkers, O.M. Comparing Methods for Measurement Error Detection in Serial 24-h Hormonal Data. J. Biol. Rhythm. 2019, 34, 347-363. [CrossRef] [PubMed]

49. Iwata, K.; Doi, A.; Miyakoshi, C. Was school closure effective in mitigating coronavirus disease 2019 (COVID-19)? Time series analysis using Bayesian inference. Int. J. Infect. Dis. 2020, 99, 57-61. [CrossRef]

50. Kasten, F.H.; Duecker, K.; Maack, M.C.; Meiser, A.; Herrmann, C.S. Integrating electric field modeling and neuroimaging to explain inter-individual variability of tACS effects. Nat. Commun. 2019, 10, 5427. [CrossRef]

51. Herrmann, C.S.; Rach, S.; Neuling, T.; Struber, D. Transcranial alternating current stimulation: A review of the underlying mechanisms and modulation of cognitive processes. Front. Hum. Neurosci. 2013, 7, 279. [CrossRef] [PubMed]

52. Antal, A.; Paulus, W. Transcranial alternating current stimulation (tACS). Front. Hum. Neurosci. 2013, 7, 317. [CrossRef] [PubMed]

53. Reato, D.; Rahman, A.; Bikson, M.; Parra, L.C. Effects of weak transcranial alternating current stimulation on brain activity-a review of known mechanisms from animal studies. Front. Hum. Neurosci. 2013, 7, 687. [CrossRef] [PubMed]

54. Thut, G.; Miniussi, C. New insights into rhythmic brain activity from TMS-EEG studies. Trends Cogn. Sci. 2009, 13, 182-189. [CrossRef]

55. Frohlich, F.; McCormick, D.A. Endogenous electric fields may guide neocortical network activity. Neuron 2010, 67, 129-143. [CrossRef]

56. Wetmore, D.Z.; Baker, S.N. Post-spike distance-to-threshold trajectories of neurones in monkey motor cortex. J. Physiol. 2004, 555, 831-850. [CrossRef] [PubMed]

57. Chen, D.; Fetz, E.E. Characteristic membrane potential trajectories in primate sensorimotor cortex neurons recorded in vivo. $J$ Neurophysiol. 2005, 94, 2713-2725. [CrossRef]

58. Wischnewski, M.; Schutter, D. After-effects of transcranial alternating current stimulation on evoked delta and theta power. Clin Neurophysiol. 2017, 128, 2227-2232. [CrossRef]

59. Whittington, M.A.; Traub, R.D.; Jefferys, J.G. Synchronized oscillations in interneuron networks driven by metabotropic glutamate receptor activation. Nature 1995, 373, 612-615. [CrossRef]

60. Rossiter, H.E.; Davis, E.M.; Clark, E.V.; Boudrias, M.H.; Ward, N.S. Beta oscillations reflect changes in motor cortex inhibition in healthy ageing. Neuroimage 2014, 91, 360-365. [CrossRef]

61. Schoffelen, J.M.; Oostenveld, R.; Fries, P. Imaging the human motor system's beta-band synchronization during isometric contraction. Neuroimage 2008, 41, 437-447. [CrossRef] [PubMed]

62. Schutter, D.J.; de Weijer, A.D.; Meuwese, J.D.; Morgan, B.; van Honk, J. Interrelations between motivational stance, cortical excitability, and the frontal electroencephalogram asymmetry of emotion: A transcranial magnetic stimulation study. Hum. Brain Mapp. 2008, 29, 574-580. [CrossRef] [PubMed]

63. Keil, J.; Timm, J.; Sanmiguel, I.; Schulz, H.; Obleser, J.; Schonwiesner, M. Cortical brain states and corticospinal synchronization influence TMS-evoked motor potentials. J. Neurophysiol. 2014, 111, 513-519. [CrossRef] [PubMed]

64. Sauseng, P.; Klimesch, W.; Gerloff, C.; Hummel, F.C. Spontaneous locally restricted EEG alpha activity determines cortical excitability in the motor cortex. Neuropsychologia 2009, 47, 284-288. [CrossRef] [PubMed]

65. Maki, H.; Ilmoniemi, R.J. EEG oscillations and magnetically evoked motor potentials reflect motor system excitability in overlapping neuronal populations. Clin. Neurophysiol. 2010, 121, 492-501. [CrossRef] [PubMed]

66. Khademi, F.; Royter, V.; Gharabaghi, A. Distinct Beta-band Oscillatory Circuits Underlie Corticospinal Gain Modulation. Cereb. Cortex 2018, 28, 1502-1515. [CrossRef] 
67. Berger, B.; Minarik, T.; Liuzzi, G.; Hummel, F.C.; Sauseng, P. EEG oscillatory phase-dependent markers of corticospinal excitability in the resting brain. Biomed. Res. Int. 2014, 2014, 936096. [CrossRef] [PubMed]

68. Iscan, Z.; Nazarova, M.; Fedele, T.; Blagovechtchenski, E.; Nikulin, V.V. Pre-stimulus Alpha Oscillations and Inter-subject Variability of Motor Evoked Potentials in Single- and Paired-Pulse TMS Paradigms. Front. Hum. Neurosci. 2016, 10, 504. [CrossRef]

69. Cappon, D.; D'Ostilio, K.; Garraux, G.; Rothwell, J.; Bisiacchi, P. Effects of $10 \mathrm{~Hz}$ and $20 \mathrm{~Hz}$ Transcranial Alternating Current Stimulation on Automatic Motor Control. Brain Stimul. 2016, 9, 518-524. [CrossRef]

70. Sanger, T.D.; Garg, R.R.; Chen, R. Interactions between two different inhibitory systems in the human motor cortex. J. Physiol. 2001, 530, 307-317. [CrossRef]

71. Schnitzler, A.; Gross, J. Normal and pathological oscillatory communication in the brain. Nat. Rev. Neurosci. 2005, 6, 285-296. [CrossRef] [PubMed]

72. Ganguly, J.; Murgai, A.; Sharma, S.; Aur, D.; Jog, M. Non-invasive Transcranial Electrical Stimulation in Movement Disorders. Front. Neurosci. 2020, 14, 522. [CrossRef]

73. Hammond, C.; Bergman, H.; Brown, P. Pathological synchronization in Parkinson's disease: Networks, models and treatments. Trends Neurosci. 2007, 30, 357-364. [CrossRef]

74. Schmidt, M.T.; Kanda, P.A.; Basile, L.F.; da Silva Lopes, H.F.; Baratho, R.; Demario, J.L.; Jorge, M.S.; Nardi, A.E.; Machado, S.; Ianof, J.N.; et al. Index of alpha/theta ratio of the electroencephalogram: A new marker for Alzheimer's disease. Front. Aging Neurosci. 2013, 5, 60. [CrossRef] [PubMed]

75. Wiethoff, S.; Hamada, M.; Rothwell, J.C. Variability in response to transcranial direct current stimulation of the motor cortex. Brain Stimul. 2014, 7, 468-475. [CrossRef] [PubMed] 